Sādhanā, Vol. 25, Part 5, October 2001, pp. 465-474. (C) Printed in India

\title{
A model with nonzero rise time for AE signals
}

\author{
M A MAJEED* and C R L MURTHY \\ Department of Aerospace Engineering, Indian Institute of Science, Bangalore \\ 560 012, India \\ *Present address: Department of Computer Science \& Engineering, Noorul \\ Islam College of Engineering, Kumaracoil, Thuckalay 629 175, India \\ e-mail: crlmurty@aero.iisc.ernet.in
}

MS received 28 November 2000; revised 16 February 2001

\begin{abstract}
Acoustic emission (AE) signals are conventionally modelled as damped or decaying sinusoidal functions. A major drawback of this model is its negligible or zero rise time. This paper proposes an alternative model, which provides for the rising part of the signal without sacrificing the analytical tractability and simplicity of the conventional model. Signals obtained from the proposed model through computer programs are illustrated for demonstrating their parity with actual AE signals. Analytic expressions for the time-domain parameters, viz., peak amplitude and rise time used in conventional AE signal analysis, are also derived. The model is believed to be also of use in modelling the output signal of any transducer that has finite rise time and fall time.
\end{abstract}

Keywords. Acoustic emission; signal parameters; signal model; exponential model; double exponential model.

\section{Introduction}

The term 'acoustic emission' (AE) refers to the stress waves produced due to transient energy release caused by irreversible deformation process in a material. Cracking of timber, tin cry, noise generated before rock and mine collapses are some of the examples of AE. Though AE literature today spreads over thousands of papers, numerous books and monographs, for quick reference bibliographies with abstracts have been brought out, e.g. by Drouillard (1979). A special technical publication by Liptai et al (1972), and reviews by Lord $(1975,1981)$ and Ono (1978) are valuable references to $\mathrm{AE}$.

A transducer mounted on the surface of the material can convert the stress waves into an electrical signal which is called 'acoustic emission signal' (AE signal). Basically, there are two types of AE signals: burst and continuous. The amplitude of an ideal burst type signal rises to a maximum value and then decays gradually. An idealized version of a burst type signal is shown in figure 1. (Henceforth, wherever we say AE signal, it means burst type signal.) This is more or less similar to AE signals obtained from experiments, which is also shown in many other references (e.g. Rao 1990, and 


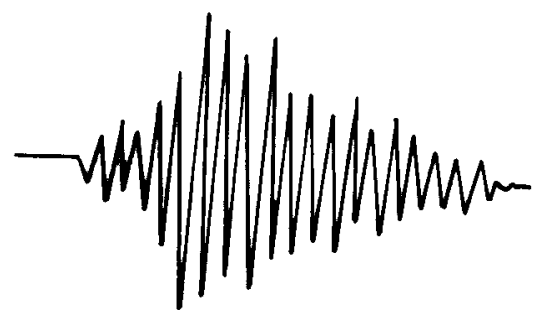

Figure 1. An idealized AE signal.

Bhat \& Murthy 1993). In the literature, such a signal is often represented by a damped sine function (e.g. Harris \& Bell 1977).

$$
X(t)=A_{0} \exp (-\alpha t) \sin 2 \Pi f_{0} t,
$$

where $A_{0}$ is the initial amplitude, $\alpha$ the damping factor and $f_{0}$ the frequency of oscillation pertaining to the resonant sensor used. Figure 2 shows a plot of this function for $A_{0}=0.5, \alpha=2.0$ and $f_{0}=50$. A major shortcoming of this model is its negligible or zero rise time (i.e. the time taken for signal to reach maximum amplitude), which is an important parameter for signal characterization and classification. The importance of rise time can be understood from papers such as those by Miller \& Mclntire (1987), Bhat \& Murthy (1993) and Majeed \& Murthy (1989). However, the damped sinusoidal model continues to be utilized even in recent literature (Teti \& Dornfeld 1989) on account of its mathematical elegance and analytical amenability. Till date, to our best knowledge,

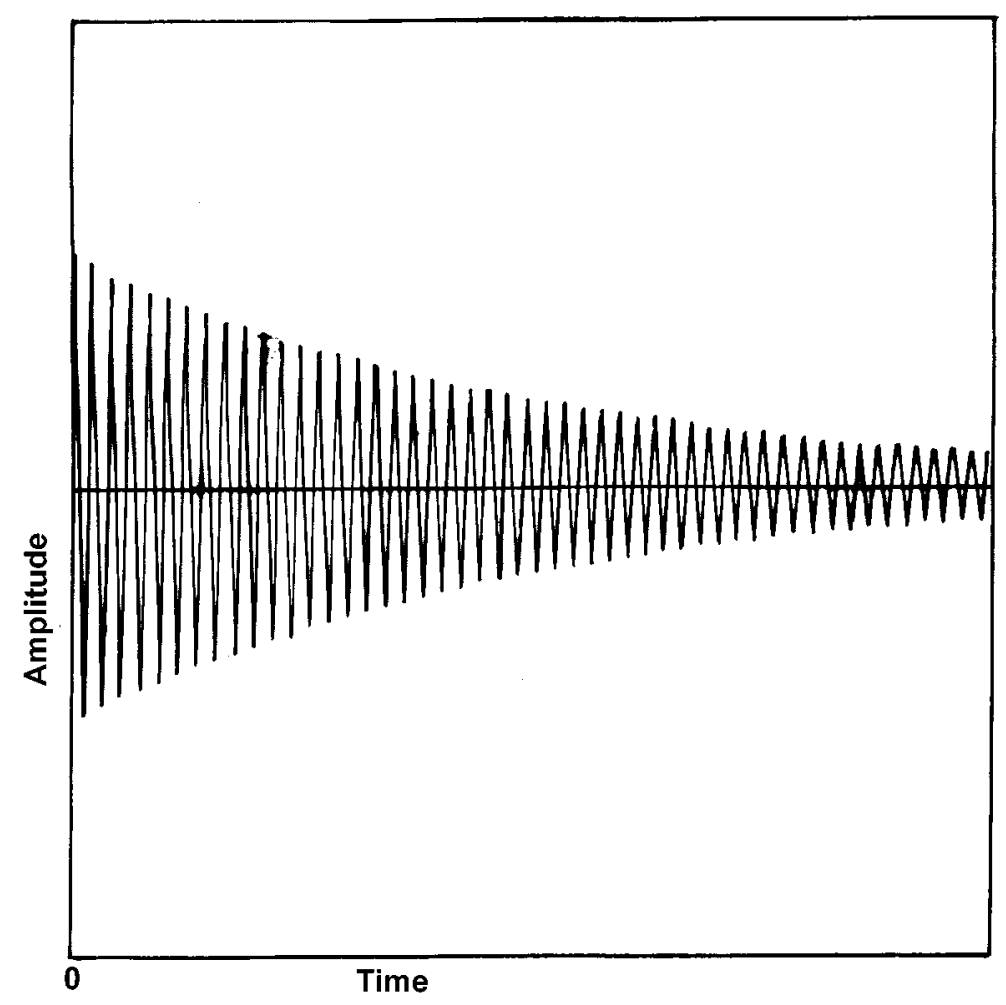

Figure 2. A damped sinusoidal signal, (1), $A_{0}=0.5, \alpha=2.0, f_{0}=50$. 
no other model has appeared in the literature. Thus, it is worthwhile to find signal models, which, while retaining these merits, can also incorporate rise time. We present such a model in the following.

\section{Proposed model}

The decaying sinusoidal model of (1) can be described in terms of communication terminology as the envelope function $A_{0} \exp (-\alpha t)$ amplitude modulating the sinusoidal signal $\sin 2 \Pi f_{0} t$. The absence of rise time in the modulated signal $A_{0} \exp (-\alpha t) \sin 2 \Pi f_{0} t$ is due to the fact that the envelope function has its maximum at the time origin. This suggests that replacement of the envelope function $A_{0} \exp (-\alpha t)$ in (1) by another function of the form shown in figure 3 will lead to the required model, i.e., model with non-zero rise time.

\subsection{Double exponential signal}

A mathematical function that can generate the graph of figure 3 is the double exponential given by

$$
e(t)=A_{0}[\exp (-\alpha t)-\exp (-\beta t)], \quad 0<\alpha<\beta .
$$

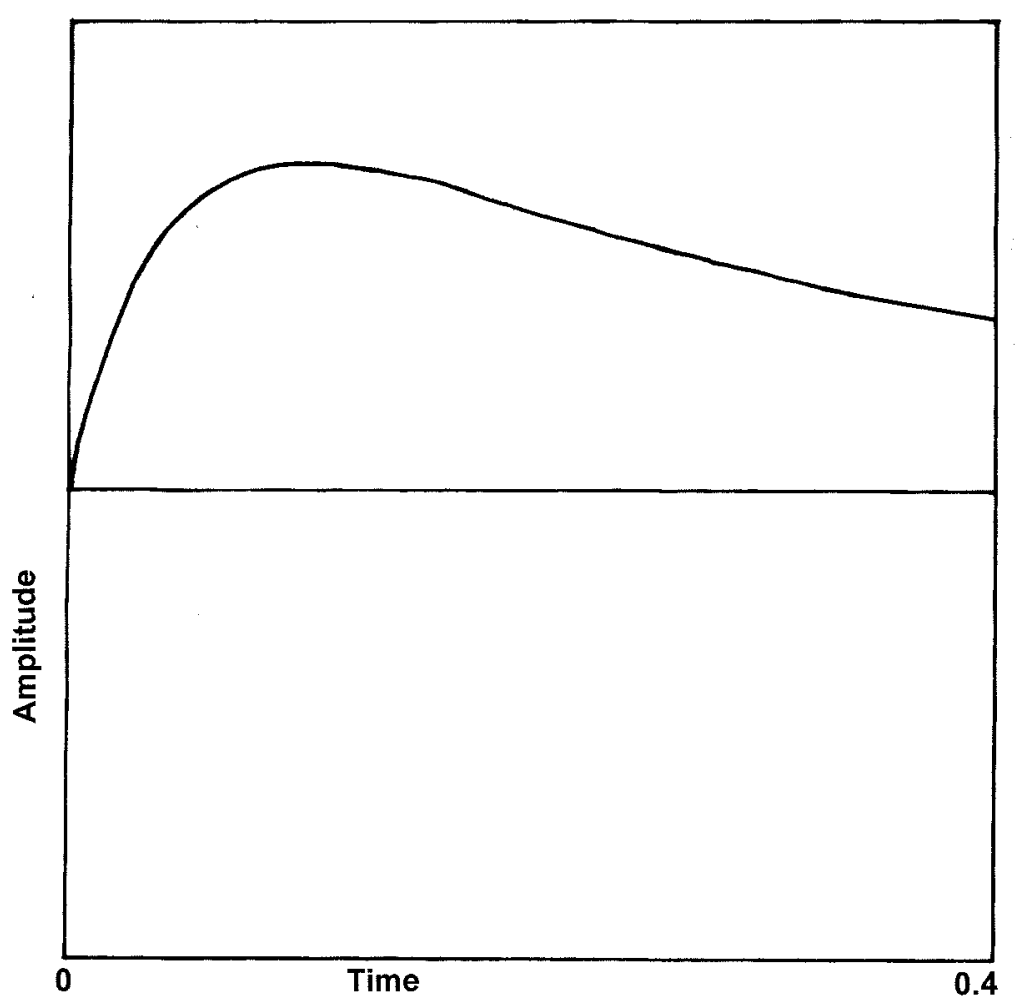

Figure 3. Envelope function with nonzero rise time, (2), $\alpha=0.001, \beta=0.01, A_{0}=1.0$. 
Figure 3 shows a plot of the above function for $\alpha=0.001$ and $\beta=0.01$. Substituting $e(t),(2)$, for $A_{0} \exp (-\alpha t)$ in (1), we get the double exponential signal

$$
V(t)=A_{0}[\exp (-\alpha t)-\exp (-\beta t)] \sin 2 \Pi f_{0} t, \quad 0<\alpha<\beta
$$

Typical signals generated by this function are shown in figures 4-7. These figures clearly demonstrate that the double exponential signal model, with rise time varying from negligible to any high value, by controlling the envelope parameters a and $b$, is a much better approximation to actual AE signals than the damped sinusoidal. In the next section, we study further the dependence of rise time and other major time-domain signal parameters on $\alpha$ and $\beta$.

Note here that the decaying sinusoidal signal is a special case of the double exponential, as

$$
\underset{\beta \rightarrow \infty}{\operatorname{Lt}}[\exp (-\alpha t)-\exp (-\beta t)] \sin 2 \Pi f_{0} t=\exp (-\alpha t) \sin 2 \Pi f_{0} t
$$

This means, if $\beta$ is very large, the present model reduces to the conventional model.

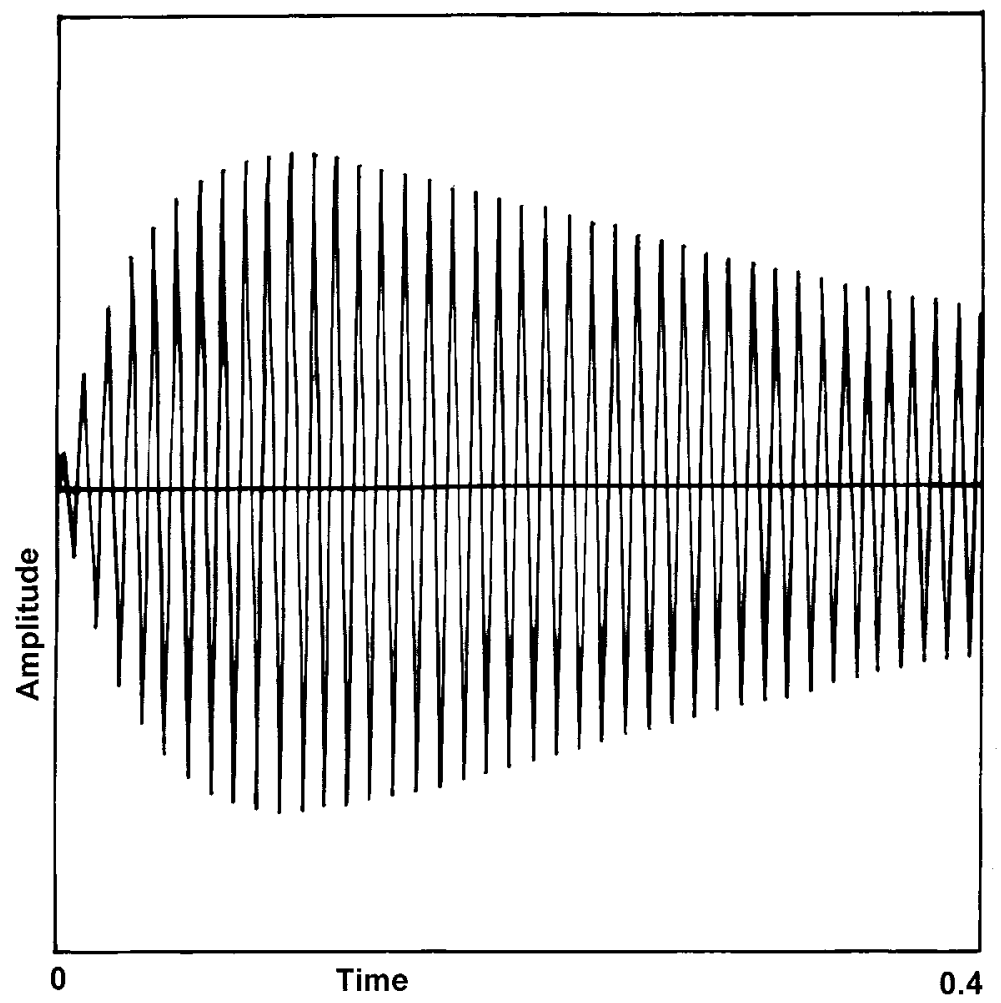

Figure 4. A double exponential signal, (3), $\alpha=0.001, \beta=0.01, A_{0}=1.0, f_{0}=100$. 


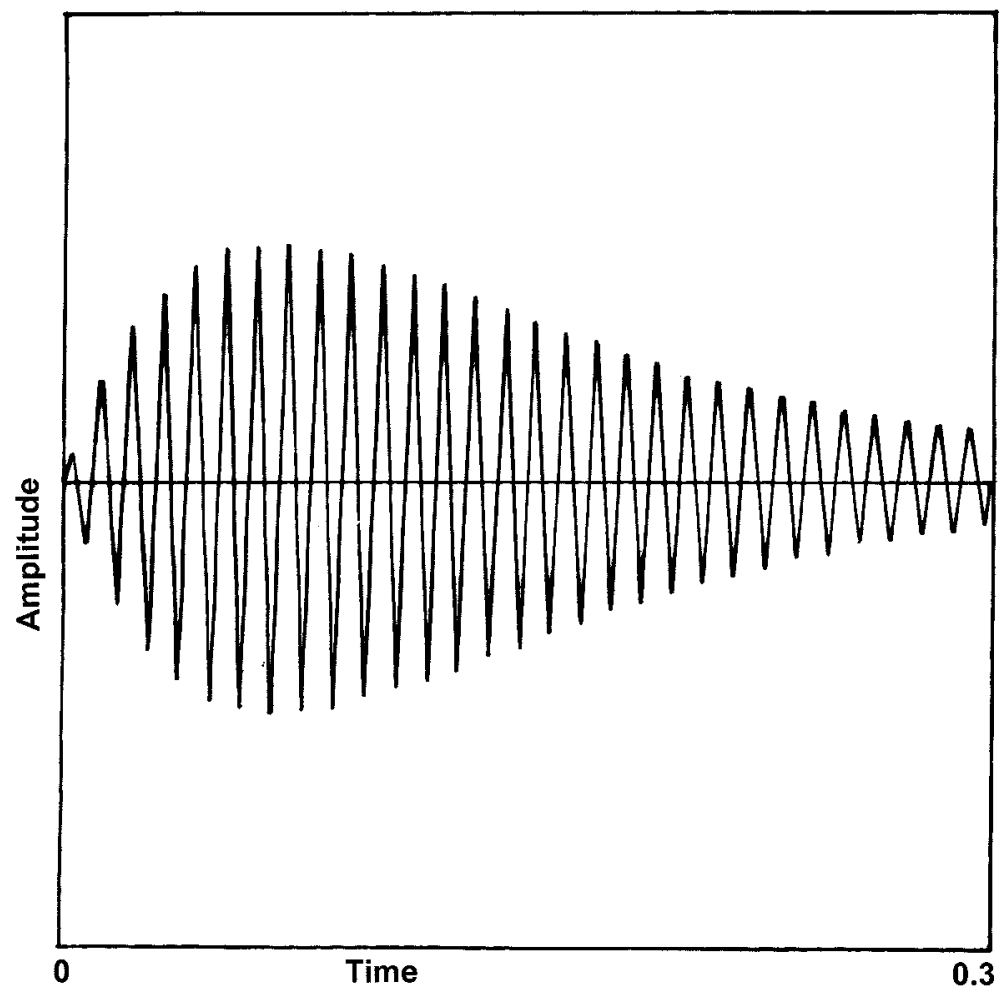

Figure 5. A double exponential signal, (3), $\alpha=10.0, \beta=20.0, A_{0}=2.0, f_{0}=100$.

\section{Time-domain parameters}

\subsection{Rise time and peak amplitude}

Rise time was defined in the introduction. Peak amplitude, as the name suggests, is the maximum value of the signal amplitude. Expressions for these parameters can be derived as follows.

From (2) we get

$$
\mathrm{d} e(t) / \mathrm{d} t=A_{0}[-\alpha \exp (-\alpha t)+\beta \exp (-\beta t)] .
$$

Rise time $\left(R_{t}{ }^{\prime}\right)$ of the envelope function $e(t)$ is obtained as follows:

$$
A_{0}[-\alpha \exp (-\alpha t)+\beta \exp (-\beta t)]=0
$$

i.e., $(\beta / \alpha)=(\exp (-\alpha t) / \exp (-\beta t))=\exp [(-\beta-\alpha) t]$,

i.e., $(\beta-\alpha) t=\log (\beta / \alpha)$,

i.e., $t=[1 /(\beta-\alpha)] \log (\beta / \alpha)$,

It is easy to show that $\mathrm{d}^{2} e(t) / \mathrm{d} t^{2}<0$ at $t=1 /(\beta-\alpha) \log (\beta / \alpha)$. Hence, rise time $R_{t}{ }^{\prime}$ of the envelope is given by

$$
R_{t}{ }^{\prime}=[1 /(\beta-\alpha)] \log (\beta / \alpha) \text {. }
$$

Also, the peak amplitude $P_{a}$ ' of the envelope is given by

$$
P_{a}{ }^{\prime}=A_{0}\left[\exp \left(-\alpha R_{t}{ }^{\prime}\right)-\exp \left(-\beta R t^{\prime}\right)\right]
$$




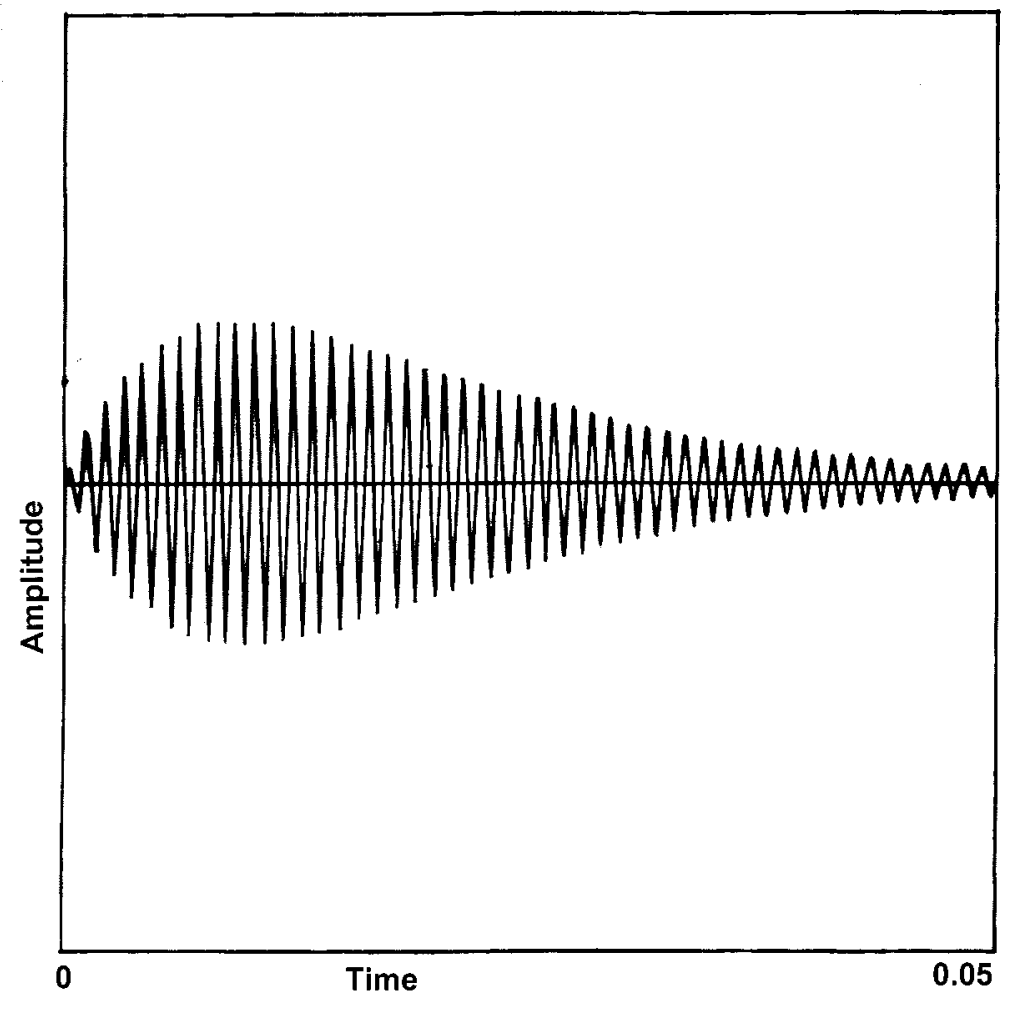

Figure 6. A double exponential signal, (3), $\alpha=100.0, \beta=110.0, f_{0}=1000$.

where $R_{t}$ ' is given by (6).

The rise time and the peak amplitude of the double exponential signal, (3), can also be represented by (6) and (7) respectively as argued below.

The local peaks of this function, (3), can be arranged into a sequence $\{V(n)\}$ as

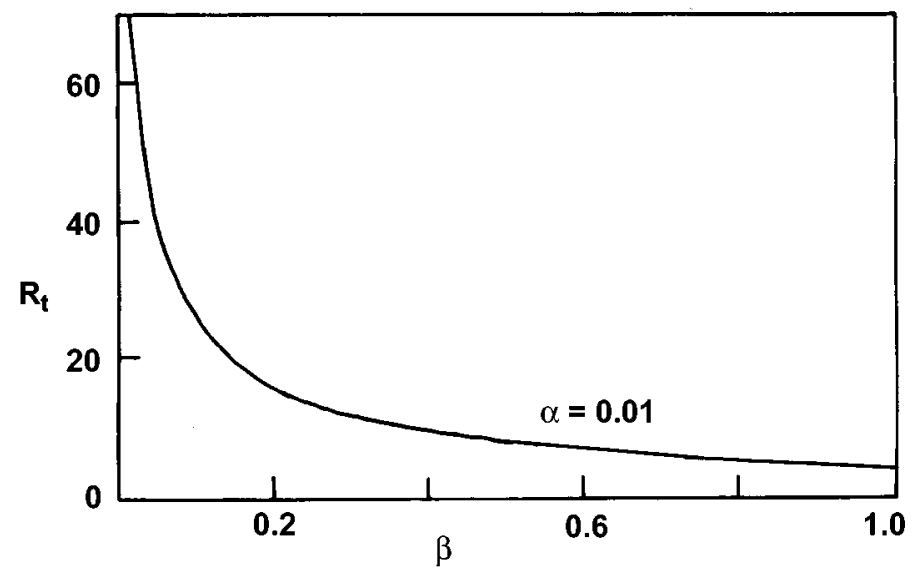

Figure 7. Variation of rise time with $\beta$ for $\alpha=0.01$. 


$$
\{V(n)\}=A_{0}\left\{\exp \left[-\alpha(2 n+1) T_{0} / 4\right]-\exp \left[-\beta(2 n+1) T_{0} / 4\right]\right\} n=0,1, \ldots, m,
$$

where $m$ is such that $V(m) \approx 0, T_{0}=1 / f_{0}$. The peak amplitude of the signal

$$
P_{a}=\max _{n}\{v(n)\} \text {. }
$$

If $f_{0}$ is such that $(2 n+1) T_{0} / 4=R_{t}$ ' for some $n$, then the rise time of the double exponential signal $R_{t}=R_{t}{ }^{\prime},(6)$, and $P_{a}=P_{a}{ }^{\prime},(7)$. Otherwise, $R_{t}$ and $R_{t}{ }^{\prime}$ satisfy the inequality

$$
\left|R_{t}{ }^{\prime}-R_{t}\right|<T_{0}=1 / f_{0}
$$

From this, we get

$$
\underset{f_{0} \rightarrow \infty}{\mathrm{Lt}} R_{t}{ }^{\prime}-R_{t}=0 \text {, i.e. } R_{t}{ }^{\prime} \rightarrow R_{t} \text { as } f_{0} \rightarrow \circ \circ .
$$

Thus if the frequency $f_{0}$ is sufficiently large, then rise time $R_{t} \approx R t^{\prime}$ as given by (6).

Also, $P_{a} \approx P_{a}{ }^{\prime}{ }^{(7)}$. $R_{t}$ is plotted in figure 8 and $P_{a}$ in figure 9 against $\beta$, keeping $\alpha$ constant. These figures show that $R_{t}$ decreases and $P_{a}$ increases with increase in $\beta$. Similar plots obtained by changing $\alpha$ and keeping $\beta$ constant are shown in figures 10 and 11 .

3.1a Rise time with respect to a threshold: In AE experiments, the rise time is usually measured with respect to a threshold. Rise time, in such cases, is measured as the time taken by the signal to reach the peak amplitude after it first crosses the threshold. Getting an exact expression for rise time (with respect to a threshold) from the double exponential model appears to be rather difficult because $t$ cannot be explicitly solved from (2). However, the time when the signal first crosses the threshold can be obtained by numerically solving the equation

$$
A_{0}[\exp (-\alpha t)-\exp (-\beta t)]=V_{t},
$$

where $V_{t}$ is the threshold voltage. The rise time (used in experiments) is the difference between this time and the rise time in (2).

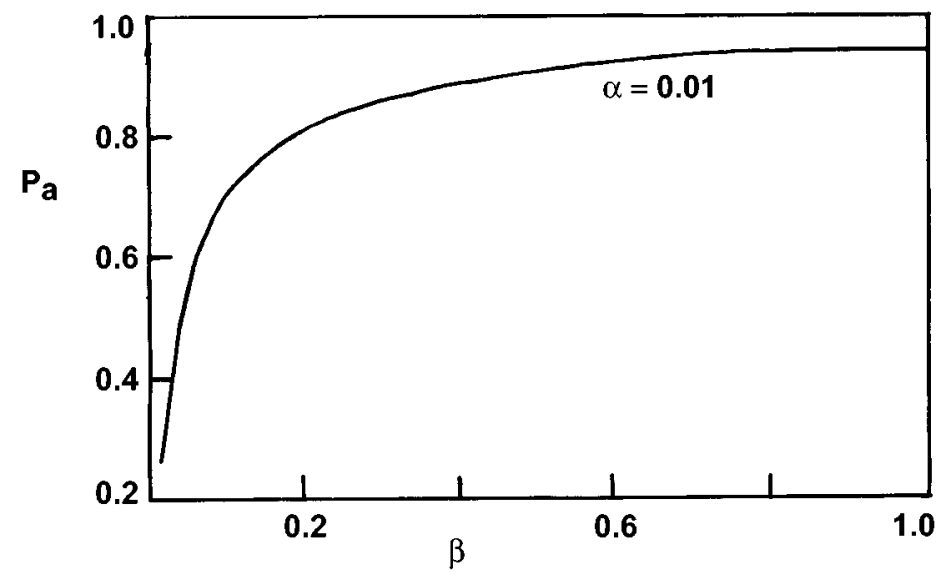

Figure 8. Variation of peak amplitude with $\beta$ for $\alpha=0.01$. 


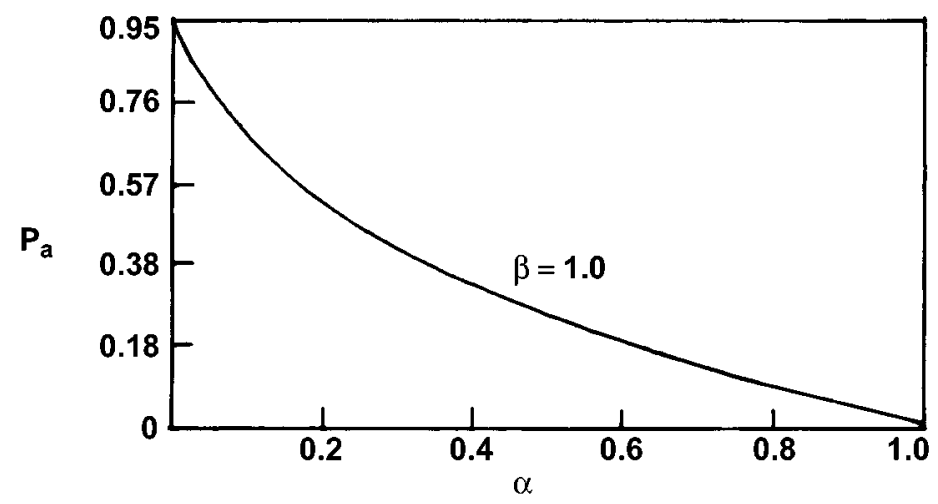

Figure 9. Variation of rise time with $\alpha$ for $\beta=1.0$.

\subsection{Ring-down count}

Ring down count is the number of times the signal crosses a preset threshold. Derivation of an exact closed-form expression for ring-down count $\left(R_{d}\right)$ appears to be impossible due to the non-invariability of the envelope function, (2). However, an approximate expression can be obtained by imposing certain conditions on the values of $\alpha, \beta$ and the threshold voltage $V_{t}$. First we assume that $\beta \gg \alpha$, which implies that there is an instant of time $t_{0}$ such that $\exp (-\beta t) \approx 0$ for all $t>t_{0}$. Under this condition, (2) becomes

$$
E(t) \approx A_{0} \exp (-\alpha t), \quad \text { for } t>t_{0} .
$$

Equation (11) can be rewritten as

$$
t \approx(1 / \alpha) \log \left(A_{0} / e(t)\right) .
$$

If $t$ ' denotes the time when $e(t)$ attains the threshold voltage $V_{t}$ then we get

$$
t^{\prime} \approx(1 / \alpha) \log \left(A_{0} / V_{t}\right),
$$

and

$$
R d=\left(f_{0} / \alpha\right) \log \left(A_{0} / V_{t}\right)
$$

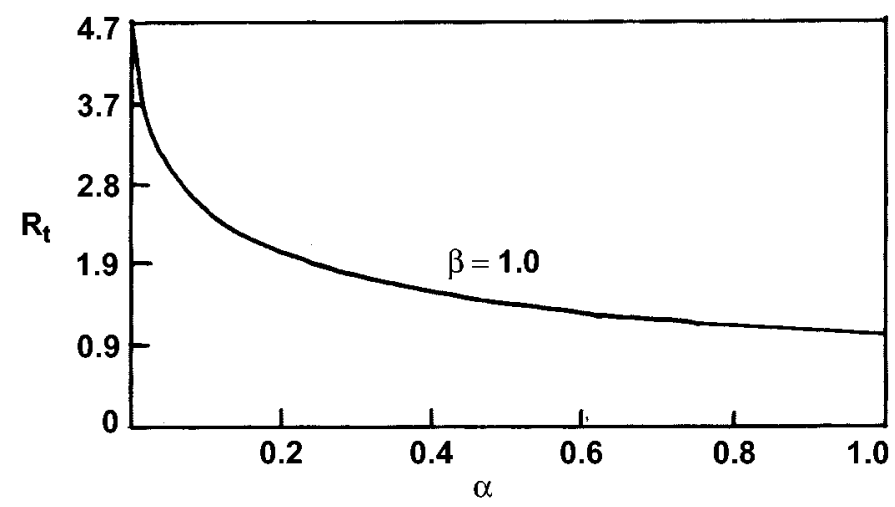

Figure 10. Variation of peak amplitude with $\alpha$ for $\beta=1.0$. 


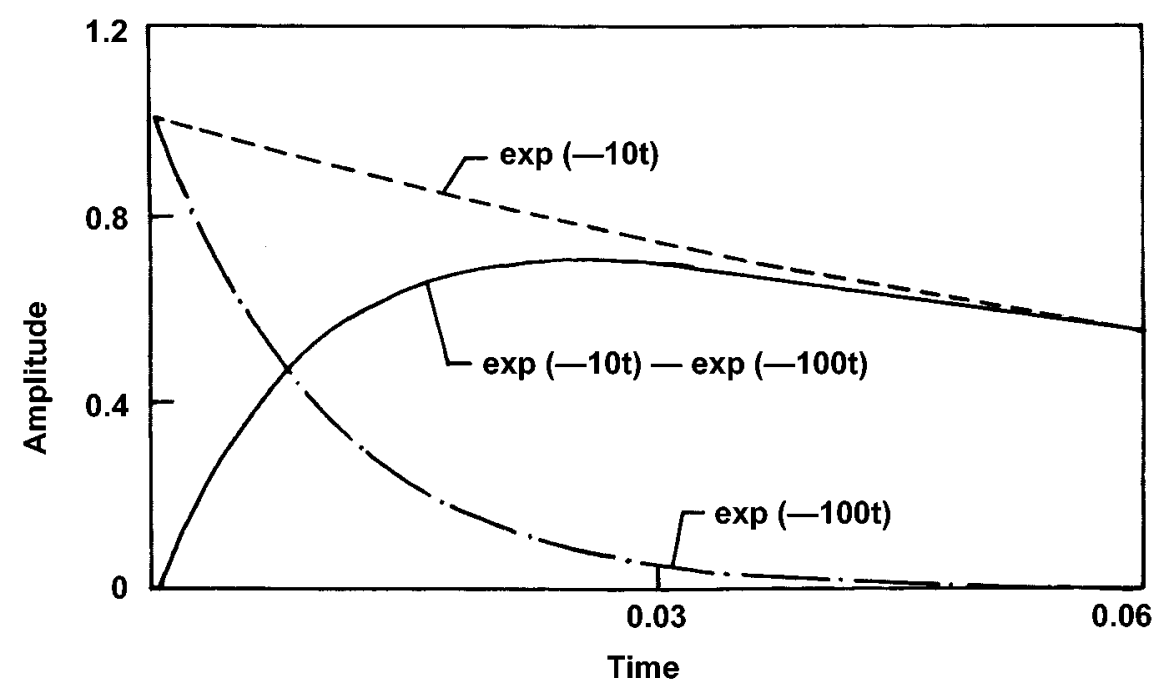

Figure 11. Approximation of double exponential by single exponential.

\section{Conclusion}

The double exponential signal model proposed in this paper is a more generalized version of the conventional exponentially decaying or single exponential model. Unlike the conventional model, the present model has the merit of possessing a rise time that is a function of the signal envelope parameters, which in turn are attributable to the source and transducer characteristics.

We thank Dr. Thomas Chacko of the Foreign Languages Section, Indian Institute of Science, for help with the language in this paper.

\section{References}

Bhat M R, Murthy C R L 1993 Fatigue damage stages in unidirectional glass-fibre-epoxy composites: identification through acoustic emission technique. Int. J. Fatigue 15: 401-405

Drouillard T F 1979 Acoustic emission - A bibliography with abstracts (New York: Plenum)

Harris D O, Bell R L 1977 The measurement and significance of energy in acoustic emission testing. Exp. Mech. 17: 347-353

Liptai R G, Harris D O, Tatro C A 1972 Acoustic emission, ASTM special technical publication 505, ASTM, Philadelphia

Lord A E Jr 1975 Acoustic emission. Physical acoustics (eds) W P Mason, R N Thurston (New York: Academic Press) vol. 11, pp 289-353

Lord A E Jr 1981 Acoustic emission - an update. Physical acoustics (New York: Academic Press) vol. 15, pp 295-360

Murthy C R L, Majeed M A, Pathak S C, Rao A K 1985 In-flight monitoring for incipient cracks in an aeroengine mount: An approach through pattern recognition. J. Acoust. Emission 4: S147-S150 
Majeed M A, Murthy C R L 1989 An efficient unsupervised pattern recognition procedure for acoustic emission signal analysis. J. Acoust. Emission 8: S16-S18

Ono K (ed.) 1978 Fundamentals of acoustic emission. Proc. Joint Meeting of Acoustic Society, Japan and America, Honolulu, Hawai (Los Angeles: Materials Department, UCLA)

Rao A K 1990 Acoustic emission and signal analysis. Defence Sci. J. 40: 55-70

Miller K R, Mclntire P (eds) 1987 Nondestructive testing handbook vol. 5, p. 28

Teti R, Dornfeld D 1989 Modelling and experimental analysis of acoustic from metal cutting. J. Eng. Ind. 111: 229-237 